\title{
Hedging Interest Rate Risk by Optimization in Banach Spaces ${ }^{1,2}$
}

\author{
A. BALBÁS ${ }^{3}$ AND R. ROMERA ${ }^{4}$
}

\begin{abstract}
This paper addresses the hedging of bond portfolios interest rate risk by drawing on the classical one-period no-arbitrage approach of financial economics. Under quite weak assumptions, several maximin portfolios are introduced by means of semi-infinite mathematical programming problems. These problems involve several Banach spaces; consequently, infinitedimensional versions of classical algorithms are required. Furthermore, the corresponding solutions satisfy a saddle-point condition illustrating how they may provide appropriate hedging with respect to the interest rate risk.
\end{abstract}

Key Words. Interest rate risk, maximin portfolio, semi-infinite programming.

\section{Introduction}

Mathematical programming has been traditionally applied in economics and engineering. However, a growing interest of applications in finance is observed. After the pioneering work of Markowitz, a large number of papers have addressed portfolio choice problems by using optimization methods. Currently, many financial market linked problems are the core of relevant optimization contributions. The objective of the present paper is to study a hedging problem that involves the term structure of interest rates (TSIR). The problem is to hedge a portfolio of multiple liabilities. The hedging instruments are coupon bonds.

\footnotetext{
${ }^{1}$ This research was partially supported by the Comunidad Autonoma de Madrid (Spain), Grant 06/HSE/0150/2004, and by MEyC (Spain), Grants BEC2000-1388-C04-03 and BEC2000-0167.

${ }^{2}$ The authors thank the unknown reviewer whose suggestions have led to significant improvements of the paper.

${ }^{3}$ Professor, Departamento de Economía de la Empresa, Universidad Carlos III de Madrid, Getafe, Madrid, Spain.

${ }^{4}$ Associate Professor, Departamento de Estadistica, Universidad Carlos III de Madrid, Getafe, Madrid, Spain; e-mail: mrromera@est-econ.uc3m.es.
} 
For the simple case of hedging a short position in a zero-coupon bond by using only long coupon bonds, it is worth noting that empirical studies show that the old-fashioned duration approach has produced better hedging strategies than those derived from more recent approaches (Ref. 1). A theoretical justification is provided in Refs. $2-5$. These papers show that the bond price convexity implies that the duration strategies are close to maximin strategies; therefore, they are robust against different changes on the TSIR and not only against parallel shifts. If one considers the more realistic problem of hedging a portfolio of long and short coupon bonds, then the convexity is lost.

We draw on the classical one-period no-arbitrage approach of financial economics and analyze the time interval between the current date and the horizon planning period $m$. The set of states of nature is a set of real functions on a time interval $[0, T], T \geq m$, and corresponds to the feasible shocks on the TSIR. The absence of arbitrage is assumed as well as the absence (in general) of a riskless asset. We deal with $n$ bonds (or more general assets) and suppose the existence of functionals $V_{j}(k(t)), j=1,2, \ldots, n$, providing a relationship between any shift $k(t)$ on the TSIR and the value at $m$ of the $j$ th asset. In this framework, we introduce the maximin strategies that play the role of hedging portfolios.

This approach allows us to work under weak hypotheses. For instance, the convexity of $V_{j}$ is not necessarily required (therefore, short-selling restrictions do not have to be imposed) and the tax effects may be incorporated. The uniqueness of the horizon planning period may be eliminated because we can take $m=0$ and assume short positions in theoretical zero coupon bonds that depend on the manager's liabilities (see Ref. 6 for a further discussion). The set of admissible shocks $k(t)$ on the TSIR contains a vast number of possibilities, since no special dynamics is given.

The level of generality forces us to consider a mathematical framework far more complex than usual in the literature. Indeed, if convexity fails, the saddlepoint characterization of maximin portfolios given in Ref. 4 does not hold. Hence, we have to modify the functional $V_{j}$ so that its convexity (even linearity) may be retrieved in some sense. This is done by identifying $V_{j}$ as a part of a new functional $E_{j}$ depending on a probability distribution and yielding the expected value at $m$ of the $j$ th security under that probability distribution. As a consequence, we deal with spaces of probability measures as well as spaces of continuous functions, $L^{2}$ spaces, and other Banach spaces.

The paper is outlined as follows. Section 2 presents notations and basic assumptions and results. Section 3 illustrates the relationships between maximin portfolios and some semi-infinite optimization problems. Section 4 summarizes a simplex-like method in Banach spaces and provides an illustrative example. Section 5 concludes the paper. 


\section{Preliminaries and Notations}

Consider $n$ arbitrary bonds $B_{j}, j=1,2, \ldots, n$, and denote by $p=$ $\left(p_{1}, p_{2}, \ldots, p_{n}\right), p_{j}>0, j=1,2, \ldots, n$, the vector of prices. Suppose that $T$ is a future date such that the bond maturities lie within the interval $[0, T]$. Suppose that $m \in[0, T]$ represents the horizon planning period and $K$ is a set of real-valued functions on $[0, T]$ whose elements are the admissible shocks on the TSIR. The portfolio composed of $q_{j}$ units of $B_{j}, j=1,2, \ldots, n$, is represented by $q=\left(q_{1}, q_{2}, \ldots, q_{n}\right) \in \mathbb{R}^{n}$ and $\sum_{j=1}^{n} p_{j} q_{j}$ is its price. If $V_{j}(k)$ is the value of $B_{j}, j=1,2, \ldots, n$, at $m$ if $k \in K$ takes place, then the real-valued function $V: \mathbb{R}^{n} \times K \rightarrow \mathbb{R}$ given by

$$
V(q, k)=\sum_{j=1}^{n} q_{j} V_{j}(k)
$$

provides the value of $q=\left(q_{1}, \ldots, q_{n}\right)$ at $m$ if $k$ takes place. Important cases of possible sets $K$ and expressions for $V(q, k)$ are given.

The expression (1) shows that the function $V$ is linear in the variable $q$. We assume that $K$ is endowed with an appropriate topology and becomes Hausdorff and compact. Moreover, $V_{j}: K \rightarrow \mathbb{R}, j=1,2, \ldots, n$, is assumed to be continuous; therefore, it follows from (1) that $V(q,-): K \rightarrow \mathbb{R}$ is also continuous in the variable $k$.

Given $q \in \mathbb{R}^{n}$, we define its guaranteed value at $m$ by

$$
\bar{V}(q)=\operatorname{Min}\{V(q, k) ; k \in K\},
$$

where $\bar{V}: \mathbb{R}^{n} \rightarrow \mathbb{R}$ is a superadditive and positively homogeneous function. That is, the properties

$$
\bar{V}\left(q+q^{\prime}\right) \geq \bar{V}(q)+\bar{V}\left(q^{\prime}\right) \quad \text { and } \quad \bar{V}(\beta q)=\beta \bar{V}(q)
$$

hold for every $\beta \geq 0$ and every $q, q^{\prime} \in \mathbb{R}^{n}$. Consequently, $\bar{V}$ is concave.

Definition 2.1. Given $Q \subset \mathbb{R}^{n}$ and $\bar{q} \in Q, \bar{q}$ is said to be $Q$-maximin if $\bar{V}(q) \leq \bar{V}(\bar{q})$ holds for every $q \in Q$.

The set $Q$ is defined by real constraints in practical applications. They may be related to budget, short-selling, or duration restictions, liabilities, and other situations.

Maximin portfolios may be very appropriate as hedging strategies because they generalize the classical concept of immunized portfolio, as proved in Ref. 5 in a model where short sales are not allowed.

The classical one-period approach of financial economics considers that the states of nature are given by probability spaces rather than Hausdorff and compact 
spaces and that future values are square-integrable functions rather than continuous ones. This fact implies some advantages, since compactness is not required and the space of square-integrable functions makes it possible to apply those properties associated only with Hilbert spaces (orthogonality, see for instance Ref. 7). Instead, the set of states of nature must be endowed with an initial probability measure and this is the reason why we have modified the general framework. We focus on very general sets of shocks and it would be difficult in practice to provide these sets with realistic probabilities. Besides, Proposition 2.1 below shows that the compactness of $K$ is a general enough hypothesis and includes most cases as the shocks of Refs. 3, 5, 6 .

Proposition 2.1. Consider two positive real numbers $\lambda_{1}, \lambda_{2}$ and suppose that $C[0, T]$ and $L^{2}[0, T]$ are the spaces of continuous and square-integrable functions on $[0, T]$. Endow $C[0, T]$ with its usual norm topology and $L^{2}[0, T]$ with the weak topology. Then:

(a) If $K_{1} \subset C[0, T]$ is composed of those continuously differentiable functions $k$ such that $|k(t)| \leq \lambda_{1}$ and $\left|k^{\prime}(t)\right| \leq \lambda_{2}$, for every $t \in[0, T]$, then $K_{1}$ is compact.

(b) If $K_{2} \subset L^{2}[0, T]$ is composed of those functions $k$ such that $|k(t)| \leq \lambda_{1}$ and $\left|k\left(t_{2}\right)-k\left(t_{1}\right)\right| \leq \lambda_{2}$ almost everywhere, then $K_{2}$ is compact.

(c) If $K_{3}$ is a closed ball of $L^{2}[0, T]$, then $K_{3}$ is compact.

\section{Proof.}

(a) The mean-value theorem permits us to prove that $K_{1}$ is equi-continuous and the Ascoli-Arzela's theorem shows that all the closed, equi-continuous, and norm-bounded subsets of $C[0, T]$ are compact; see Ref. 8 for further details on the applied results.

(b) Suppose that $k$ is in $K_{2}$. We have that

$$
\|k\|_{2}=\left[\int_{0}^{T} k(t)^{2} d t\right]^{0.5} \leq\left(\lambda_{1}^{2} T\right)^{0.5}=\lambda_{1} T^{0.5},
$$

and the set of feasible shocks is norm-bounded. Thus, the Alaoglu theorem implies that set of feasible shocks is weak-compact if it is weak-closed. Since this set is clearly convex, the Hahn-Banach theorem shows that it is sufficient to prove that this set is closed. Hence, assume that the sequence $\left(k_{\theta}\right)_{\theta \in \mathrm{N}} \subset K_{2}$ tends to $k$ in the norm of $L^{2}[0, T], \mathbb{N}$ being the set of natural numbers. Then, classical measure theory shows that a subsequence converges almost everywhere to $k$; therefore, $k \in K_{2}$, since $|k(t)| \leq \lambda_{1}$ and $\left|k\left(t_{2}\right)-k\left(t_{1}\right)\right| \leq \lambda_{2}$ almost everywhere.

(c) It is an immediate consequence of the Alaoglu theorem. 
As usual, $\mathcal{C}(K)$ denotes the Banach space of real-valued and continuous functions on $K$ endowed with the supremum norm. The Riesz representation theorem establishes that $\mathcal{M}(K)$ is the dual space of $\mathcal{C}(K), \mathcal{M}(K)$ being the Banach space of regular $\sigma$-additive measures on the Borel $\sigma$-algebra of $K$. The set of nonnegative measures

$$
\mathcal{M}_{+}(K)=\{\mu \in \mathcal{M}(K) ; \mu \geq 0\}
$$

and the set of probability measures

$$
\mathcal{P}(K)=\left\{\mu \in \mathcal{M}_{+}(K) ; \mu(K)=1\right\}
$$

are important subsets of $\mathcal{M}(K)$; see Refs. 8, 9 for further details.

If $k \in K$, we consider its associated Dirac delta that concentrates all the mass on $k$, i.e., the probability measure $\delta_{k}$ with $\delta_{k}(k)=1$. In some sense, $k$ may be identified with $\delta_{k}$; therefore, $K$ may be understood as a subset of $\mathcal{P}(K)$. Consider $E: \mathbb{R}^{n} \times \mathcal{M}(K) \longrightarrow \mathbb{R}$ given by

$$
E(q, \mu)=\int_{K} V(q, k) d \mu(k),
$$

for $q \in \mathbb{R}^{n}$ and $\mu \in \mathcal{M}(K)$. $E$ is a bilinear extension of $V$, since

$$
E\left(q, \delta_{k}\right)=V(q, k),
$$

for every $q \in \mathbb{R}^{n}$ and every $k \in K$.

Proposition 2.2. If $q \in \mathbb{R}^{n}$, then $\bar{V}(q)=\operatorname{Min}\{E(q, \mu) ; \mu \in \mathcal{P}(K)\}$.

Proof. The inequality

$$
\bar{V}(q) \geq \operatorname{Inf}\{E(q, \mu) ; \mu \in \mathcal{P}(K)\}
$$

follows trivially from (2) and (3). Besides, (2) implies that

$$
V(q, k) \geq \bar{V}(q),
$$

for every $k \in K$; thus,

$$
E(q, \mu)=\int_{K} V(q, k) d \mu(k) \geq \int_{K} \bar{V}(q) d \mu(k)=\bar{V}(q)
$$

holds for every $\mu \in \mathcal{P}(K)$. Hence,

$$
\operatorname{Inf}\{E(q, \mu) ; \mu \in \mathcal{P}(K)\} \geq \bar{V}(q) .
$$




\section{Semi-Infinite Programming and Maximin Portfolios}

This section is devoted to analyze some semi-infinite optimization problems leading to maximin strategies. Thus, consider $Q \subset \mathbb{R}^{n}$ and the problem

$$
\begin{array}{ll}
\text { Max } & y, \\
\text { s.t. } & \sum_{j=1}^{n} q_{j} V_{j}(k) \geq y, \quad \forall k \in K, \\
& q \in Q,
\end{array}
$$

with $q \in \mathbb{R}^{n}$ and $y \in \mathbb{R}$ being the decision variables. Problem (4) provides a useful tool when computing maximin portfolios, since we have the following result whose proof is omitted because it is quite simple.

Proposition 3.1. Given $Q \subset \mathbb{R}^{n}$ and $\bar{q} \in Q$, we have that $\bar{b}$ is $Q$-maximin if and only if there exists $\bar{y} \in \mathbb{R}$ such that $(\bar{q}, \bar{y})$ solves (4). In the affirmative case, the equality $\bar{y}=\bar{V}(\bar{q})$ holds.

Let us consider Problem (5) and Problem (6) below

$$
\begin{array}{ll}
\text { Max } & y, \\
\text { s.t. } & \sum_{j=1}^{n} q_{j} V_{j}(k) \geq y, \quad \forall k \in K, \\
& q \in Q, \\
& \sum_{j=1}^{n} p_{j} q_{j} \leq \bar{\theta}, \\
\text { Max } & \sum_{j=1}^{n} p_{j} q_{j}, \\
& \sum_{j=1}^{n} q_{j} V_{j}(k) \geq \theta^{*}, \quad \forall k \in K, \\
\text { s.t. } & q \in Q .
\end{array}
$$

$\bar{\theta}$ and $\theta^{*}$ being arbitrary constants and $(q, y) \in \mathbb{R}^{n} \times \mathbb{R}$ and $q \in \mathbb{R}^{n}$ being the decision variables.

Proposition 3.1 points out that (5) yields the $Q^{\prime}$-maximin portfolio where $Q^{\prime}$ is composed of those portfolios of $Q$ whose total price is not larger than $\bar{\theta}$. 
In practice, $\bar{\theta}$ may represent the capital to invest. Special attention may merit the self-financing case $\bar{\theta}=0$ that arises frequently when a manager is hedging liabilities of a given fund. On the other hand, Problem (6) allows agents to fix the amount of money $\theta^{*}$ to be guaranteed and to minimize the amount to invest.

Proposition 3.2. Consider a subset $Q$ of $\mathbb{R}^{n}$ and two arbitrary real numbers $\bar{\theta}$ and $\theta^{*}$. Suppose that $q^{*}$ is the unique solution of (6) and take $\bar{\theta}=\sum_{j=1}^{n} p_{j} q_{j}^{*}$.

Then, $\left(q^{*}, \bar{V}\left(q^{*}\right)\right)$ solves (5) and $q^{*}$ is $\left\{q \in Q ; \sum_{j=1}^{n} p_{j} q_{j}^{*} \leq \bar{\theta}\right\}$-maximin.

Proof. If $\left(q^{*}, \bar{V}\left(q^{*}\right)\right)$ does not solve (5), then take $(q, y) \in Q \times \mathbb{R}$ such that

$$
\begin{aligned}
& \sum_{j=1}^{n} p_{j} q_{j} \leq \sum_{j=1}^{n} p_{j} q_{j}^{*}, \\
& \sum_{j=1}^{n} q_{j} V_{j}(k) \geq y>\bar{V}\left(q^{*}\right),
\end{aligned}
$$

for every $k \in K$. Since $q^{*}$ is (6)-feasible, one has that $\bar{V}\left(q^{*}\right) \geq \theta^{*}$ and $q$ is (6)feasible due to (8). Thus, $q^{*}$ is not the unique solution of (6) owing to (7).

The previous proposition may be established also if the uniqueness of $q^{*}$ is replaced by another appropriate assumption. Moreover, as is shown in Theorem 3.1 , it may be extended in the convex case. We focus on this situation with $\theta^{*}=1$. So, consider a convex set $Q \subset \mathbb{R}^{n}$ and the problem

$$
\begin{array}{ll}
\text { Min } & \sum_{j=1}^{n} p_{j} q_{j}, \\
\text { s.t. } & \sum_{j \in=}^{n} q_{j} V_{j}(k) \geq 1, \quad \forall k \in K, \\
& q \in \mathbb{R}
\end{array}
$$

$q=\left(q_{1}, \ldots, q_{n}\right)$ being the decision variable. Problem (9) provides the minimum price of those portfolios belonging to $Q$ and guaranteeing one dollar.

Problem (9) is semi-infinite because the first constraint is valued in the space $\mathcal{C}(K)$. Following the approach of Ref. 9, the dual variable belongs to the dual space $\mathcal{M}(K)$ and the dual problem admits a maximin expression in terms of the Lagrangian function. The dual problem becomes

$$
\operatorname{Max}_{\mu \in \mathcal{M}_{1}(K)}\left[\operatorname{In} f_{q \in Q}\left\{\int_{K} d_{\mu}(k)+\sum_{j=1}^{n} q_{j}\left(p_{j}-\int_{K} V_{j}(k) d \mu(k)\right)\right\}\right],
$$


$\mu \in \mathcal{M}(K)$ being the decision variable. The absence of a duality gap between (9) and (10) is not guaranteed, since we are in the face of infinite-dimensional problems (see Ref. 9). Thus, hereafter we will assume additional conditions.

Assumption A. There exists $\alpha>0$ such that $V_{1}(k) \geq \alpha$ for every $k \in K$.

Condition C. The set $Q$ of Problems (9) and (10) is convex. Moreover, there are $\beta_{2}, \ldots, \beta_{n} \in \mathbb{R}$ such that $q=\left(\beta, \beta_{2}, \ldots \beta_{n}\right) \in Q$ for every $\beta>0$.

Condition $\mathrm{C}$ is clearly satisfied if $(1,0,0, \ldots 0) \in Q$ and $Q$ is a convex cone. Conditions $\mathrm{A}$ and $\mathrm{C}$, along with arbitrage arguments, solve the duality gap between (9) and (10). In fact, first of all, Problem (9) is feasible due to A and C. Furthermore, (9) is also bounded, since an optimal value equal to $-\infty$ would imply the existence of portfolios with negative price guaranteeing one dollar at least, providing investors with an arbitrage opportunity. Besides, the duality gap between (9) and (10) vanishes and (10) is solvable because A and $\mathrm{C}$ lead to the existence of $q=\left(\beta, \beta_{2}, \ldots \beta_{n}\right) \in Q$ such that $V(q, k)>1$ for every $k \in K$; therefore, the Slater qualification holds (Ref. 9). Finally, the optimal value $M_{Q}$ of (9) and (10) cannot be negative, since, once again, $M_{Q}<0$ would imply arbitrage. These properties are summarized in the statement below.

Proposition 3.3. Problems (9) and (10) are feasible and bounded and do not generate a duality gap. Problem (10) is solvable and the optimal value of both problems is positive if achieved in (9).

Theorem 3.1. Saddle-Point Theorem. Consider an arbitrary convex cone $Q$ and a positive amount of money $C>0$. Define

$$
Q_{C}=\left\{q \in Q ; \sum_{j=1}^{n} p_{j} q_{j} \leq C\right\} .
$$

Assume that $\mu^{*}$ solves (10), suppose that (9) attains its optimal value $M_{Q}$, and take $R_{Q}=1 / M_{Q}$. Then, the following assertions are equivalent:

(a) $q^{*} \in Q$ solves (9).

(b) $\bar{q}=C R_{Q} q^{*}$ is $Q_{C}$-maximin.

(c) $\left(C R_{Q} q^{*}, R_{Q} \mu^{*}\right)$ is a saddle point of $E$ in $Q_{C} \times \mathcal{P}(K)$, i.e.,

$$
E\left(q, R_{Q} \mu^{*}\right) \leq E\left(C R_{Q} q^{*}, R_{Q} \mu^{*}\right) \leq E\left(C R_{Q} q^{*}, \mu\right),
$$

for every $q \in Q_{C}$ and every $\mu \in \mathcal{P}(K)$. 
Proof. (a) $\Rightarrow$ (c) Suppose that $\mu$ is a probability measure. Since $q^{*}$ is (9)-feasible, we have that

$$
V\left(q^{*}, k\right) \geq 1, \quad \text { for every } k \in K .
$$

Therefore,

$$
E\left(q^{*}, \mu\right)=\int_{K} V\left(q^{*}, k\right) d \mu(k) \geq \int_{K} d \mu(k)=1
$$

and

$$
E\left(q^{*}, \mu\right) \geq E\left(q^{*}, R_{Q} \mu^{*}\right)
$$

will be proved if

$$
E\left(q^{*}, R_{Q} \mu^{*}\right)=1 .
$$

Suppose that we prove the inequality

$$
\sum_{j=1}^{n} p_{j} q_{j}^{*} \geq E\left(q^{*}, \mu^{*}\right)
$$

Then, bearing in mind that $\bar{V}\left(q^{*}\right) \geq 1\left[q^{*}\right.$ is (9)-feasible] and that the price of $q^{*}$ and the integral of $d \mu^{*}$ must equal $M_{Q}$, we have that

$$
\begin{aligned}
M_{Q} & =\sum_{j=1}^{n} p_{j} q_{j}^{*} \geq E\left(q^{*}, \mu^{*}\right) \\
& =\int_{K} V\left(q^{*}, k\right) d \mu^{*}(k) \\
& \geq \bar{V}\left(q^{*}\right) \int_{K} d \mu^{*}(k) \\
& \geq \int_{K} d_{\mu^{*}}(k)=M_{Q} .
\end{aligned}
$$

Hence, all the previous terms must be equal and (11) follows after multiplying the whole expression by $R_{Q}$. Finally, let us see that (12) is fulfilled because $\mu^{*}$ is (10)-feasible. Indeed, according to (10),

$$
\operatorname{Inf}\left\{\int_{K} d \mu^{*}(k)+\sum_{j=1}^{n} q_{j}\left(p_{j}-\int_{K} V_{j}(k) d \mu^{*}(k)\right) ; q \in Q\right\}>-\infty .
$$

Bearing in mind that $Q$ is a cone,

$$
\sum_{j=1}^{n} q_{j}\left(p_{j}-\int_{K} V_{j}(k) d \mu(k)\right) \geq 0,
$$


for every $q \in Q$, since otherwise $q$ could be multiplied by $\lambda \rightarrow \infty$ and (13) would fail. The expression (14) implies that

$$
\sum_{j=1}^{n} q_{j} p_{j} \geq E\left(q, \mu^{*}\right) .
$$

In order to prove the remainder inequality of the saddle-point condition, notice again that

$$
E\left(q, \mu^{*}\right) \leq \sum_{j=1}^{n} q_{j} p_{j}
$$

for every $q \in Q$. In particular, if the price of $q$ is not greater than $C$, then

$$
E\left(q, R_{Q} \mu^{*}\right) \leq R_{Q} C,
$$

whereas the equality

$$
E\left(C R_{Q} q^{*}, R_{Q} \mu^{*}\right)=R_{Q} C
$$

follows from (11).

(c) $\Rightarrow$ (b) Proposition 2.2 and the inequality

$$
E\left(C R_{Q} q^{*}, R_{Q} \mu^{*}\right) \leq E\left(C R_{Q} q^{*}, \mu\right)
$$

clearly imply that

$$
\bar{V}\left(C R_{Q} q^{*}\right)=E\left(C R_{Q} q^{*}, R_{Q} \mu^{*}\right) .
$$

Besides, from Proposition 2.2,

$$
\bar{V}(q) \leq E\left(q, R_{Q} \mu^{*}\right) \leq E\left(C R_{Q} q^{*}, R_{Q} \mu^{*}\right),
$$

for every $q \in Q_{C}$.

(b) $\Rightarrow$ (a) Since $C R_{Q} q^{*}$ is $Q_{C}$-maximin, we have that $C R_{Q} q^{*} \in Q_{C}$. Thus, the price of $q^{*}$ cannot be larger than $M_{Q}$; consequently, it is sufficient to prove that $q^{*}$ is (9)-feasible. Let $q$ be a solution of (9). The price of $q$ equals $M_{Q}$ and $\bar{V}(q) \geq 1$. Hence, the price of $C R_{Q} q$ equals $C$ and, bearing in mind that $\bar{V}$ is positively homogeneous and $C R_{Q} q^{*}$ is $Q_{C}$-maximin, we have that

$$
1 \leq \bar{V}\left(q^{*}\right)
$$


because

$$
\begin{aligned}
C R_{Q} & \leq C R_{Q} \bar{V}(q) \\
& =\bar{V}\left(C R_{Q} q\right) \\
& \leq \bar{V}\left(C R_{Q} q^{*}\right) \\
& =C R_{Q} \bar{V}\left(q^{*}\right) .
\end{aligned}
$$

Remark 3.1. If $Q$ is a convex cone, then Problem (10) becomes

$$
\begin{array}{ll}
\operatorname{Max} & \int_{K} d \mu(k), \\
\text { s.t. } & \sum_{j=1}^{n} p_{j} q_{j} \geq E(q, \mu), \quad \forall q \in Q, \\
& \mu \geq 0 .
\end{array}
$$

Theorem 3.1 illustrates that the maximin portfolio and the probability measure $R_{Q} \mu^{*}$ are given by the solutions of (9) and (15), which can be derived from a family of complementary slackness conditions. Thus, $q^{*}$ and $\mu^{*}$ solve

$$
\begin{aligned}
& \sum_{j=1}^{n} q_{j} V_{j}(k) \geq 1, \quad \forall k \in K, \\
& \sum_{j=1}^{n} q_{j}^{\prime} p_{j} \geq E\left(q^{\prime}, \mu\right), \quad \forall q^{\prime} \in Q, \\
& q \in Q \quad \text { and } \quad \mu \geq 0, \\
& \sum_{j=1}^{n} q_{j} V_{j}(k)=1, \quad \forall k \in S p(\mu), \\
& \sum_{j=1}^{n} q_{j} p_{j}=E(q, \mu),
\end{aligned}
$$

$q \in \mathbb{R}^{n}$ and $\mu \in \mathcal{M}(K)$ being unknowns and $S p(\mu)$ denoting the support of $\mu$.

\section{Applying a Semi-Infinite Simplex-Like Method}

Propositions 3.2 and 3.3 and Theorem 3.1 have justified already the interest of semi-infinite programming algorithms when computing maximin portfolios 
in practice. Furthermore, depending on the properties of $Q$, the involved optimization problems may be also convex or linear. The analysis of semi-infinite programming algorithms has been deeply addressed in the literature and is beyond our scope. However, since Theorem 3.1 and Remark 3.1 point out the relevance of Problems (9) and (15), we present some of their features. In this line, Lemmas 4.1 and 4.2 below justify the interest of studying the set $\operatorname{ext}\left(\mathcal{F}_{d}\right)$ of extreme points of the (15)-feasible set $\mathcal{F}_{d}$. Both proofs are omitted, since similar results are presented in Ref. 10.

Lemma 4.1. Let $Q$ be a convex cone. For every $\varepsilon>0$, there exists an extreme point $\mu_{\varepsilon}$ of the (15)-feasible set such that

$$
\int_{K} d \mu_{\varepsilon}(k) \geq M_{Q}-\varepsilon
$$

Lemma 4.2. $\operatorname{ext}(\{\mu \in \mathcal{M}(K) ; \mu \geq 0, \mu(K) \leq 1\})=\{0\} \cup\left\{\delta_{k} ; k \in K\right\}$.

As usual, define

$$
q+Q=\left\{q+q^{\prime} \in \mathbb{R}^{n} ; q^{\prime} \in Q\right\}, \quad \text { for every } Q \subset \mathbb{R}^{n} \text { and every } q \in \mathbb{R}^{n} .
$$

Then, recall that

$$
L_{Q}=\{q \in Q ; q+Q=Q\}
$$

is a linear space for any arbitrary closed convex cone $Q \subset \mathbb{R}^{n}$. If $L_{Q}^{\top}$ represents the orthogonal space of $L_{Q}$, then the recession cone of $L_{Q}^{\top} \cap Q$ is the set

$$
\tilde{L}_{Q}^{\top}=\left\{q \in L_{Q}^{\top} \cap Q ; q+L_{Q}^{\top} \cap Q \subset L_{Q}^{\top} \cap Q\right\} .
$$

It may be proved that $L_{Q}^{\top}$ is a closed pointed convex cone. Moreover, $\tilde{L}_{Q}^{\top}$ has a finite basis if there exists $\left\{q^{1}, q^{2}, \ldots, q^{s}\right\} \subset \tilde{L}_{Q}^{\top}$ such that every element of $\tilde{L}_{Q}^{\top}$ takes the form $\sum_{i=1}^{s} x_{i} q^{i}$, with $x_{1}, x_{2}, \ldots, x_{s}$ being nonnegative scalars. In such a case, we will say that the dimension of $\tilde{L}_{Q}^{\top}$ is $\operatorname{dim}\left(\tilde{L}_{Q}^{\top}\right)=s$, if there are no basis of $\tilde{L}_{Q}^{\top}$ whose number of elements is smaller than $s$ (Ref. 11).

Remark 4.1. Assume now that $Q$ is a closed convex cone,

$$
\operatorname{dim}\left(\tilde{L}_{Q}^{\top}\right)=s, \operatorname{dim}\left(L_{Q}\right)=r .
$$

Let $\left\{q^{1}, q^{2}, \ldots, q^{s}\right\}$ be a basis of $\tilde{L}_{Q}^{\top}$ and let $\left\{q^{s+1}, q^{s+2}, \ldots, q^{s+r}\right\}$ be a basis of $L_{Q}$. The representation theorem of closed convex sets (Ref. 11) ensures that $Q$ coincides with the set of points $q$ satisfying $q=\sum_{i=1}^{s+r} \lambda_{i} q^{i}$, where $\lambda_{1}, \lambda_{2}, \ldots, \lambda_{s+r} \in \mathbb{R}$ and $\lambda_{1}, \lambda_{2}, \ldots, \lambda_{s} \geq 0$. Then, once the slack variables are 
introduced, it is immediate to prove that (15) is equivalent to

$$
\begin{array}{ll}
\operatorname{Max} & \int_{K} d \mu(k), \\
\text { s.t. } & E\left(q^{i}, \mu\right)+x_{i}=p^{i}, \quad i=1,2, \ldots, s, \\
& E\left(q^{i}, \mu\right)=p^{i}, \quad i=s+1, s+2, \ldots, s+r, \\
& \mu \in \mathcal{M}(K) \quad \text { and } \quad \mu \geq 0, \\
& x_{1}, x_{2}, \ldots, x_{s} \geq 0, \quad \text { in } \mathbb{R},
\end{array}
$$

where $x_{1}, x_{2}, \ldots, x_{s}$ and $\mu$ are the decision variables and

$$
p^{i}=\sum_{j=1}^{n} p_{j} q_{j}^{i}
$$

is the price of $q^{i}, i=1,2, \ldots, s+r$. It is clear that there exists a bijection between the (15)-feasible set and the (17)-feasible one that preserves the extreme points. Thus, if there is no confusion, we will denote both sets by $\mathcal{F}_{d}$.

If one combines Lemma 4.2 and some results of Ref. 12 concerning the extreme points of several sets of probability measures, then one gets the following theorem.

Theorem 4.1. Under the assumptions of Remark 4.1, if $(\mu, x) \in \mathcal{M}(K) \times$ $\mathbb{R}^{s}$ is a extreme point of $\mathcal{F}_{d}$, then there exist $\left\{k_{1}, \ldots, k_{t}\right\} \subset K$ and $\left\{\alpha_{1}, \ldots, \alpha_{t}\right\} \subset$ $\mathbb{R}$ such that $\alpha_{i} \geq 0, i=1,2, \ldots, t, \sum_{i=1}^{t} \alpha_{i} \leq M_{Q}$, and $\mu=\sum_{i=1}^{t} \alpha_{i} \delta_{k_{i}}$. Furthermore, if $t^{\prime}$ is the cardinal of $\operatorname{Sp}(x)=\left\{i ; x_{i} \neq 0\right\}$, then $t+t^{\prime} \leq s+r$.

Remark 4.2. Simplex-Like Algorithm. Next, we summarize the simplexlike method allowing us to solve (17). We do not justify the steps of the algorithm, since they follow easily from Ref. 10 . We merely adapt the method to our particular problem.

Step 1. Take an initial nondegenerate (i.e., $t+t^{\prime}=s+r$, with the notations of the previous theorem) $(\mu, x) \in \operatorname{ext}\left(\mathcal{F}_{d}\right)$.

Step 2. Let $\rho$ be the number of strictly positive slack variables of $(\mu, x)$. To make things easier, suppose that $x_{1}, \ldots, x_{\rho}>0$ and $x_{\rho+1}=\cdots=x_{s}=0$. Take $\operatorname{Sp}(\mu)=\left\{k_{\rho+1}, \ldots, k_{s+r}\right\}$. Let $B$ be the (basic) square matrix of dimension $s+r$,

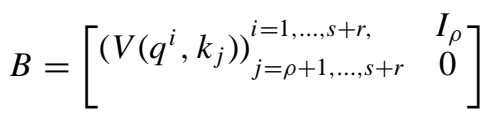


where $i$ indicates the row, $j$ indicates the column, and $I_{p}$ denotes the identity matrix whose dimension is $\rho$. The matrix $B$ is regular. Define $\Gamma_{k}=(1, \ldots, 1,0, \ldots, 0) B^{-1}\left[\begin{array}{c}\left(V\left(q^{i}, k\right)\right)_{i=1}^{\rho} \\ \left(V\left(q^{i}, k\right)\right)_{i=\rho+1}^{s+r}\end{array}\right], \quad$ for every $k \in K$, $\Gamma_{i}=(1, \ldots, 1,0, \ldots, 0) B^{-1} E_{i}^{t}, \quad$ for $i=1,2, \ldots, s$, $E_{i}^{t}$ being the transpose of $E_{i}=(0,0, \ldots, 0,1,0, \ldots, 0), \quad i=1,2, \ldots, s+r$.

It is important to remark that $\Gamma_{k}-1=0, \quad$ if $k \in \operatorname{Sp}(\mu)$, $\Gamma_{i}=0, \quad$ if $i \in \operatorname{Sp}(x)$.

Compute $\Gamma=\operatorname{Min}\left(\left\{\Gamma_{k}-1 ; k \in K\right\} \cup\left\{\Gamma_{i}, i=1,2, \ldots, s\right\}\right)$.

We have that $\Gamma$ cannot be strictly positive. If $\Gamma=0$, then $(\mu, x)$ solves (17).

Step 3. Case 1. Assume that $\Gamma=\Gamma_{k_{0}}-1<0$. Then, $k_{0} \notin S p(\mu)$. Take

$$
\begin{aligned}
& \left(\gamma_{\rho+1}, \ldots \gamma_{s+r}, \gamma_{1}, \ldots, \gamma_{\rho}\right)^{t}=B^{-1}\left(V\left(q^{i}, k_{0}\right)_{i=1}^{s+r}\right), \\
& \gamma=\operatorname{Min}\left[\{ \mu ( k _ { j } ) , x _ { i } / \gamma _ { j } ; j = \rho + 1 , \ldots s + r , \gamma _ { j } > 0 \} \cup \left\{x_{i} / \gamma_{i} ;\right.\right. \\
& \left.\left.\quad=1, \ldots, \rho, \gamma_{j}>0\right\}\right], \\
& \left(\bar{\mu}\left(k_{\rho+1}\right), \ldots, \bar{\mu}\left(k_{s+r}\right), \bar{x}_{1}, \ldots, \bar{x}_{\rho}\right)^{t} \\
& =\left(\mu\left(k_{\rho+1}\right), \ldots, \mu\left(k_{s+r}\right), x_{1}, \ldots, x_{\rho}\right)^{t} \\
& -\gamma\left(\gamma_{\rho+1}, \ldots \gamma_{s+r}, \gamma_{1}, \ldots, \gamma_{\rho}\right)^{t},
\end{aligned}
$$

and $\bar{\mu}\left(k_{0}\right)=\gamma$. We have that $(\bar{\mu}, \bar{x}) \in \operatorname{ext}\left(\mathcal{F}_{d}\right)$ and the objective value has been improved according to

$$
\int_{K} d \bar{\mu}(k)=\int_{K} d \mu+\bar{\mu}\left(k_{0}\right)\left(1-\Gamma_{k_{0}}\right) .
$$

Step 3. Case 2. Assume that $\Gamma=\Gamma_{\theta}<0$. Then, $\theta$ does not belong to $\operatorname{Sp}(x)$. Take $\left(\gamma_{\rho+1}, \ldots \gamma_{s+r}, \gamma_{1}, \ldots, \gamma_{\rho}\right)^{t}=B^{-1} E_{\theta}^{t}$,

$\gamma$ as (18) indicates, $\bar{x}_{\theta}=\gamma$, and $\left(\bar{\mu}\left(k_{\rho+1}\right), \ldots, \bar{\mu}\left(k_{s+r}\right), \bar{x}_{1}, \ldots, \bar{x}_{\rho}\right)^{t}$ 
as (19) indicates. Then, $(\bar{\mu}, \bar{x}) \in \operatorname{ext}\left(\mathcal{F}_{d}\right)$ and the objective value improves according to

$$
\int_{K} d \bar{\mu}(k)=\int_{K} d \mu-\bar{x}_{\theta} \Gamma_{\theta} .
$$

Step 4. Let $(\bar{\mu}, \bar{x})$ play the role of $(\mu, x)$ and go back to Step 2 .

Example 4.1. Two particular cases arise when short sales on the available bonds are imposed $\left(Q=\mathbb{R}_{+}^{n}\right)$ or omitted $\left(Q=\mathbb{R}^{n}\right)$. In the first situation $L_{Q}=\{0\}$ and $\tilde{L}_{Q}^{T}=\mathbb{R}_{+}^{n}$, whereas $L_{Q}=\mathbb{R}^{n}$ and $\tilde{L}_{Q}^{T}=\{0\}$ in the second one. Thus, Problem (17) becomes Problem (20) or Problem (21) below

$$
\begin{array}{ll}
\operatorname{Max} & \int_{K} d \mu(k), \\
\text { s.t. } & \int_{K} V_{j}(k) d \mu(k)+x_{j}=p_{j}, \quad j=1,2, \ldots, n, \\
& \mu \geq 0, x_{1}, x_{2}, \ldots, x_{s} \geq 0,
\end{array}
$$

or

$$
\begin{array}{ll}
\operatorname{Max} & \int_{K} d \mu(k), \\
\text { s.t. } & \int_{K} V_{j}(k) d \mu(k)=p_{j}, \quad j=1,2, \ldots, n, \\
& \mu \geq 0 .
\end{array}
$$

We have applied the simplex algorithm in order to compute the maximin portfolio in a market where there are 6 available bonds with annual coupon equal to 0.1 dollars, principal value equal to 1 dollar, and maturity in $3,4,5,6,7,8$ years respectively. For simplicity's sake, we assume a flat initial TSIR with a constant value equal to $10 \%$. Then,

$$
V(q, k)=\sum_{r=1}^{N} c_{r} \exp \left(\int_{t_{r}}^{m}(k(t)+0.1) d t\right),
$$

where $\left\{c_{1}, c_{2}, \ldots, c_{n}\right\}$ is the set of coupons paid by $q$ at $\left\{t_{1}, t_{2}, \ldots, t_{N}\right\}$ respectively. We have taken $m=5$ years and $K$ is the set of shocks introduced in Ref. 5 and analyzed in Proposition 2.1b. We have selected the parameter $\lambda_{2}=0.05=5 \%$ and it may be proved that the final result does not depend on $\lambda_{1}$.

Under short sale restrictions the solution was: Buy the bonds with maturity in $6(81.37 \%$ of the total investment) and 7 years $(18.63 \%)$. The guaranteed amount 
is $96.1 \%$ of the initial capitalization rate $R$ between zero and five years. For the unconstrained problem, we obtained

$$
q=(-32.41 \%,-13.60 \%, 146.76 \%,-0.27 \%,-46.84 \%, 47.65 \%),
$$

guaranteeing $98.77 \%$ of $R$.

\section{Conclusions}

This paper has addressed the existence, properties, and computation of maximin portfolios in a hedging problem involving the TSIR. We have used the one-period approach of financial economics and have taken the shock on the TSIR as the state of nature. Once those functionals yielding the securities final value are extended to abstract spaces, a general theory on optimization in banach spaces may apply. From a financial viewpoint, the approach may be very useful to traders and researches, since it works under weak assumptions. For instance, convexity and short-selling restrictions do not have to be imposed, tax effects may be incorporated, the uniqueness of the horizon planning period may be relaxed, interest rate-linked derivatives could be involved, and the set of admissible shocks on the interest rates contains a vast number of possibilities because there are no dynamic assumptions about them.

\section{References}

1. Bierwag, G. O., Fooladi, I., and Roberts, G. S., Designing an Immunized Portfolio: Is M-Squared the Key?, Journal of Banking and Finance, Vol. 17, pp. 1147-1170, 1993.

2. BIERWaG, G.O., and Khang, C., An Immunization Strategy is a Maxmin Strategy, Journal of Finance, Vol. 37, pp. 379-389, 1979.

3. FonG, H. G., and VASICEK, O. A., A Risk Minimizing Strategy for Portfolio Immunization, Journal of Finance, Vol. 39, pp. 1541-1546, 1984.

4. PRISMAN, E.Z., Immunization as a Maximin Strategy: New Look, Journal of Banking and Finance, Vol. 10, pp. 491-509, 1986.

5. BAlbÁs, A., and Ibá̃̃EZ, A., When Can You Immunize a Bond Portfolio? Journal of Banking and Finance, Vol. 22, pp. 1571-1595, 1998

6. Bowden, R.J., Generalizing Interest Rates Duration with Directional Derivatives, Management Science, Vol. 43, pp. 586-595, 1997.

7. LuenBerger, D. G., Pricing a Nontradeable Asset and Its Derivatives, Journal of Optimization Theory and Applications, Vol. 3, pp. 465-487, 2004.

8. HoRvÁtH, J., Topological Vector Spaces and Distributions, Vol. 1, Addison Wesley, Reading, Massachusetts, 1966.

9. LUENBERGER, D. G., Optimization by Vector Spaces Methods, John Wiley and Sons, New York, NY, 1969. 
10. Anderson, E.J., and NAsh, P., Linear Programming in Infinite-Dimensional Spaces, John Wiley and Sons, New York, NY, 1987.

11. Holmes, R. B., Geometrical Functional Analysis and Its Applications, Graduate Texts in Mathematics, Springer Verlag, New York, NY, Vol. 24, 1975.

12. WinkLeR, G., Extreme Points of Moment Sets, Mathematics of Operations Research, Vol. 13, pp. 581-587, 1988. 